\title{
Associação entre dislipidemia, dados sociodemográficos, hábitos sedentários e alimentação inadequada em escolares do sul do Brasil
}

\author{
Association between dyslipidemia, sociodemographic data, \\ sedentary behavior and improper feeding in schoolchildren \\ from southern of Brazil
}

Janaína Domingues Corrêa, ${ }^{1}$ Cleiton Bertollo, ${ }^{1}$ Ana Paula Sehn,, Deise Graziela Kern, ${ }^{1}$ Letícia Welser, Cristiane Fernanda da Silva, ${ }^{1}$ Gilmar Fernando Weis, ${ }^{1}$ Cézane Priscila Reuter, ${ }^{1}$ Miria Suzana Burgos ${ }^{1}$ 'Universidade de Santa Cruz do Sul (UNISC), Santa Cruz do Sul, RS, Brasil.

Recebido em: 16/02/2017 / Aceito em: 09/03/2017 / Publicado em: 31/03/2017

cpreuter@hotmail.com

\section{RESUMO}

A dislipidemia, importante fator de risco para doenças cardiovasculares, está cada vez mais presente na infância e adolescência. Identificar os fatores associados é fundamental para elaboração de estratégias de saúde pública. Objetivo: verificar possível associação entre dislipidemia com variáveis sociodemográficas, atividades sedentárias e hábitos alimentares em escolares de um município do Sul do Brasil. Método: foram avaliados 1.243 crianças e adolescentes, sendo 563 do sexo masculino, da rede pública e privada, da zona urbana e rural do município de Santa Cruz do Sul, RS. Para avaliação de dislipidemia, considerou-se a presença de alteração em pelo menos um parâmetro do perfil lipídico (triglicerídeos, colesterol total e frações HDL e LDL). As variáveis sociodemográficas, as atividades sedentárias e os hábitos alimentares foram avaliados por questionário. O teste de corrida/caminhada de 9 minutos foi utilizado para avaliar a aptidão cardiorrespiratória. Resultados: a dislipidemia associa-se somente com variáveis sociodemográficas. Assim, a prevalência de dislipidemia é $9 \%$ maior na região centro $(p=0,030)$ e em escolas da rede municipal (RP: 1,18; $p=0,002$ ) e estadual (RP: 1,$11 ; p=0,023)$. Não foi observada relação com hábitos sedentários e ao pouco consumo de frutas/ verduras. Considerações finais: a identificação das variáveis associadas com a dislipidemia pode auxiliar na elaboração de estratégias de saúde pública.

Palavras-chave: Dislipidemias; Fatores de Risco; Estilo de Vida; Criança; Adolescente.

\section{ABSTRACT}

Dyslipidemia is an important risk factor for cardiovascular diseases and is increasingly present in childhood and adolescence. Identifying the associated factors is fundamental for the elaboration of public health strategies. Objective: to verify the possible association between dyslipidemia with sociodemographic variables, sedentary behavior and eating habits in schoolchildren from a municipality in Southern Brazil. Method: 1,243 children and adolescents were evaluated of which 563 were male, of public and private schools from the urban and rural areas of Santa Cruz do Sul, Brazil. Dyslipidemia was evaluated through the presence of alteration in at least one parameter of the lipid profile (triglycerides, total cholesterol and HDL and LDL fractions). Sociodemographic variables, sedentary activities and eating habits were assessed by questionnaire. The 9-minute run/walk test was used to assess cardiorespiratory fitness. Results: dyslipidemia was associated only with sociodemographic variables. Thus, the prevalence of dyslipidemia is $9 \%$ higher in the central region $(p=$ 0.030) and in municipal schools (RP: 1.18, $p=0.002$ ) and state schools (RP: 1.11; $p=0.023$ ). No relation was observed with sedentary habits and the low intake of fruits/vegetables. Closing remarks: the identification of the variables associated with dyslipidemia can help in the elaboration of public health strategies.

Keywords: Dyslipidemia; Risk Factors; Lifestyle; Children; Adolescents. 


\section{INTRODUÇÃO}

O desenvolvimento de novas pesquisas pode auxiliar o profissional da área de saúde na prevenção da dislipidemia infantil. ${ }^{1}$ Para tanto, o dever do profissional de Educação Física consiste em orientar e fornecer respostas para uma melhora das condições de saúde. Podemos nos referir que uma conduta saudável envolve boa alimentação, desenvolvimento físico e mental, sendo o foco principal o cuidado humano; assim, devemos trabalhar com corpo e mente em busca de bons hábitos, sendo esta uma caminhada constante de novas alternativas para resolução de problemas. ${ }^{2}$

Em busca de novos conhecimentos, cresce o número pesquisas com crianças e adolescentes para investigar a prevalência de dislipidemia, a qual tem se tornado uma epidemia nas últimas três décadas. ${ }^{3-6} \mathrm{~A}$ alimentação adequada, com redução do consumo de refrigerantes e doces, bem como a estimulação da prática de atividade física, diminuindo o sedentarismo, faz com que nossas crianças tenham um desenvolvimento saudável, podendo evitar patologias. ${ }^{7}$ Existe uma relação com a obesidade infanto-juvenil e as doenças cardiovasculares futuras. ${ }^{8}$

Obtendo um controle em suas rotinas, para que haja uma dieta rica em frutas e verduras e redução de alimentos gordurosos e refrigerantes, além da prática de atividades físicas diárias, são estratégias importantes para a diminuição de futuros riscos à saúde. ${ }^{4}$ Segundo Lachtermacher, ${ }^{7}$ podemos prevenir muitas doenças através do cuidado com a alimentação, evitando alimentos com gorduras saturadas, embutidos, excesso de sódio e doces. Zheng et al. ${ }^{9}$ defendem que a atividade física é um importante fator de prevenção e auxilia no controle da dislipidemia em crianças e adolescentes.

Devemos prevenir a obesidade infantil com medidas adequadas, assim como a prescrição de dieta na infância desde o início da vida, além da adoção de programas de educação que possam ser aplicados no nível primário de saúde e nas escolas. É de consenso que a obesidade infantil vem aumentando significativamente e desencadeando várias complicações na infância e na vida adulta. ${ }^{1}$ Dentre essas complicações estão à dislipidemia e síndrome metabólica, cada vez mais comum entre crianças e adolescentes, com excesso de peso e obesidade. ${ }^{10}$

Sabendo que a dislipidemia é um importante fator de risco para doenças cardiovasculares ${ }^{11}$ e que sua prevalência tende a aumentar com o excesso de peso e idade; com isso aumenta o risco de agravos e suas complicações. ${ }^{12}$ Os impactos da dislipidemia sobre as estruturas cardiovasculares podem ser reversíveis na infância, sendo uma fase importante e estratégica para adquirir hábitos saudáveis, sendo levados para toda a vida, evitando futuros problemas cardíacos, cada vez mais presente entre os adultos, estes uma das principais causas de morte entre a população adulta. ${ }^{13,14}$

Desta forma, o presente estudo tem como objetivo verificar possível associação de dislipidemia com variáveis sociodemográficas, atividades sedentárias e hábitos alimentares em escolares de um município do Sul do Brasil.

\section{MÉTODO}

São sujeitos do presente estudo transversal 1.243crianças e adolescentes, de 7 a 17 anos, sendo 563 do sexo masculino, da rede pública (estadual e municipal) e privada, da zona urbana e rural do município de Santa Cruz do Sul - RS. O presente estudo faz parte de uma pesquisa mais ampla, desenvolvida na Universidade de Santa Cruz do Sul (UNISC), denominada "Avaliação de indicadores bioquímicos de saúde de escolares usando espectroscopia no infravermelho, polimorfismos, saúde bucal e fatores relacionados ao estilo de vida: um estudo em Santa Cruz do Sul - Fase II". Previamente à coleta de dados, o estudo foi encaminhado ao Comitê de Ética em Pesquisa com Seres Humanos da Universidade de Santa Cruz do Sul, sob protocolo número 2959-11. Os pais ou responsáveis autorizaram a participação do escolar, mediante envio do Termo de Consentimento Livre e Esclarecido assinado.

Para avaliação do perfil lipídico (triglicerídeos, colesterol total e colesterol HDL), foi realizada coleta sanguínea no Laboratório de Bioquímica do Exercício da Universidade de Santa Cruz do Sul (UNISC), respeitando um jejum de 12 horas. As amostras de soro foram analisadas no equipamento automatizado Miura One (I.S.E., Roma, Itália), utilizando kits comerciais da marca Kovalent. Os valores do colesterol LDL foram obtidos através da equação de Friedwald, Levy e Fredrickson..$^{15}$ Para a classificação do perfil lipídico, foi utilizada a classificação do National Heart, Lung, and Blood Institute. ${ }^{16}$ Para avaliação de dislipidemia, considerou-se a presença de alteração em pelo menos um parâmetro do perfil lipídico.

Os hábitos sedentários foram avaliados através do tempo de tela dispendido pelo escolar (TV, vídeo game e computador), autoreferido pelo escolar, através de questionário utilizado na pesquisa de Burgos et al. ${ }^{17}$ Posteriormente, os dados obtidos foram classificados em: 1) pouco tempo em frente à tela $(<2$ horas diárias) e 2) muito tempo em frente à tela ( $\geq 2$ horas diárias), conforme estabelecido pela Academia Americana de Pediatria $^{18}$. Adicionalmente, foi avaliada a aptidão cardiorrespiratória (APCR), através do teste de corrida/ caminhada de 9 minutos, o qual foi aplicado em pista previamente demarcada na UNISC. Os escolares foram orientados ao uso de calçado adequado e utilização de roupa leve para a realização do teste. À distância percorrida, em metros, foi registrada e posteriormente foram utilizados os pontos de corte estabelecidos pelo Projeto Esporte Brasil, ${ }^{19}$ classificando os escolares em aptos (bons níveis de APCR) e inaptos (baixos níveis de APCR).

A avaliação do consumo de frutas, hortaliças e hábitos alimentares foi realizada por questionário utilizado na pesquisa de Burgos et al. ${ }^{17}$ adaptado de Barros e Nahas, ${ }^{20}$ que consiste na identificação da frequência semanal destes alimentos.

Para a análise dos dados, utilizou-se o programa estatístico SPSS v. 23.0 (IBM, Armonk, NY, EUA). A associação entre a variável desfecho (dislipidemia) com as variáveis preditoras (variáveis sociodemograficas, hábitos sedentários e alimentares) foi testada através da regressão de Poisson, utilizando a razão de prevalência (RP) e intervalos de confiança (IC) para 95\%. Para todas as análises, foram considerados significativos os valores de $p<0,05$.

\section{RESULTADOS}

Identificou-se, no presente estudo, que é elevado o 
percentual de escolares com dislipidemia $(41,7 \%)$, com baixos níveis de aptidão cardiorrespiratória (52,0\%), que nunca ou somente às vezes consomem frutas e verduras $(77,7 \%)$, que dispendem mais de duas horas em frente à tela da TV ou computador/videogame $(56,7 \%)$ e que comem assistindo TV (38,3\%) (Tabela 1).

Tabela 1 - Características descritivas dos sujeitos.

\begin{tabular}{|c|c|}
\hline & n (\%) \\
\hline \multicolumn{2}{|l|}{ Nível socioeconômico } \\
\hline$A-B$ & $587(55,4)$ \\
\hline C & $443(41,8)$ \\
\hline D-E & $30(2,8)$ \\
\hline \multicolumn{2}{|l|}{ Região de moradia } \\
\hline Rural & $301(28,4)$ \\
\hline Centro & $266(25,1)$ \\
\hline Periferia & $493(46,5)$ \\
\hline \multicolumn{2}{|l|}{ Rede de ensino } \\
\hline Particular & $77(7,3)$ \\
\hline Municipal & $409(38,6)$ \\
\hline Estadual & $574(54,1)$ \\
\hline \multicolumn{2}{|l|}{ Dislipidemia } \\
\hline Não & $618(58,3)$ \\
\hline Sim & $442(41,7)$ \\
\hline \multicolumn{2}{|l|}{ Aptidão cardiorrespiratória } \\
\hline Normal & $509(48,0)$ \\
\hline Indicador de risco & $551(52,0)$ \\
\hline \multicolumn{2}{|c|}{ Prática de atividade física/esportiva } \\
\hline Sim & $681(64,2)$ \\
\hline Não & $379(35,8)$ \\
\hline \multicolumn{2}{|l|}{ Consumo de frutas e verduras } \\
\hline Sempre/quase sempre & $239(22,5)$ \\
\hline Nunca/às vezes & $821(77,5)$ \\
\hline \multicolumn{2}{|l|}{ Tempo de tela* } \\
\hline Até duas horas diárias & $459(43,3)$ \\
\hline Mais de duas horas diárias & $601(56,7)$ \\
\hline \multicolumn{2}{|l|}{ Come enquanto assiste TV } \\
\hline Não & $159(15,0)$ \\
\hline Às vezes & $495(46,7)$ \\
\hline Sim & $406(38,3)$ \\
\hline
\end{tabular}

Observa-se, na tabela 2, que a dislipidemia mostrou associação somente com variáveis sociodemográficas. Dessa forma, a prevalência de dislipidemia é $9 \%$ maior na região centro e em escolas da rede municipal (RP: 1,$18 ; p=0,002$ ) e estadual (RP: 1,$11 ; p=0,023$ ). Não foi observada relação com hábitos sedentários e ao pouco consumo de frutas/verduras.

\section{DISCUSSÃO}

No presente estudo, observou-se que é elevada a prevalência de dislipidemia nos escolares avaliados (41,7\%), sendo corroborado por estudo realizado em Belém, no Pará, com escolares de 7 a 14 anos, o qual identificou $48,1 \%$ de dislipidemia nos sujeitos analisados. ${ }^{21}$ Já, em Salvador, Bahia, a prevalência encontrada em escolares de 6 a 19 anos, foi menor $(25,5 \%){ }^{22}$

Com relação aos fatores associados à dislipidemia, constatamos que esta condição é mais prevalente entre os escolares do centro (RP: 1,$09 ; p=0,030$ ) e da rede pública de ensino, tanto em escolas municipais (RP: 1,18;
Tabela 2 - Razão de prevalência bruta para variáveis associadas à dislipidemia em crianças e adolescentes. Santa Cruz do Sul, RS

\begin{tabular}{|c|c|c|}
\hline & RP (IC 95\%) & $\mathbf{P}$ \\
\hline \multicolumn{3}{|l|}{ Nível socioeconômico } \\
\hline$A-B$ & 1 & \\
\hline C & $1,00(0,96-1,05)$ & 0,941 \\
\hline D-E & $0,96(0,84-1,09)$ & 0,505 \\
\hline \multicolumn{3}{|l|}{ Região } \\
\hline Rural & 1 & \\
\hline Centro & $1,09(1,01-1,17)$ & 0,030 \\
\hline Periferia & $1,05(1,00-1,11)$ & 0,076 \\
\hline \multicolumn{3}{|l|}{ Rede de ensino } \\
\hline Particular & 1 & \\
\hline Municipal & $1,18(1,06-1,32)$ & 0,002 \\
\hline Estadual & $1,11(1,02-1,22)$ & 0,023 \\
\hline \multicolumn{3}{|l|}{ Aptidão cardiorrespiratória } \\
\hline Normal & 1 & \\
\hline Indicador de risco & $1,04(0,99-1,08)$ & 0,098 \\
\hline \multicolumn{3}{|c|}{ Prática de atividade física/esportiva } \\
\hline Sim & 1 & \\
\hline Não & $1,02(0,97-1,06)$ & 0,478 \\
\hline \multicolumn{3}{|l|}{ Consumo de frutas e verduras } \\
\hline Sempre/quase sempre & 1 & \\
\hline Nunca/às vezes & $0,99(0,94-1,04)$ & 0,675 \\
\hline \multicolumn{3}{|l|}{ Tempo de tela* } \\
\hline Até duas horas diárias & 1 & \\
\hline Mais de duas horas diárias & $0,98(0,94-1,02)$ & 0,309 \\
\hline \multicolumn{3}{|l|}{ Come enquanto assiste TV } \\
\hline Não & 1 & \\
\hline Às vezes & $0,99(0,93-1,05)$ & 0,753 \\
\hline Sim & $1,00(0,93-1,05)$ & 0,901 \\
\hline
\end{tabular}

$p=0,002$ ), quanto em estaduais (RP: 1,$11 ; p=0,023$ ). Não foi observada relação com o nível socioeconômico, o que também foi observado no estudo realizado com crianças de Amsterdam, na Holanda, avaliando cada componente do perfil lipídico separadamente. ${ }^{23}$ Porém, no estudo de Silva e colaboradores, ${ }^{24}$ não se observou diferença significativa para a dislipidemia em relação ao local de sua moradia; no entanto, obteve-se maior prevalência de dislipidemia em meninas.

Além disso, não foram observadas diferenças significativas entre dislipidemia e hábitos sedentários, como a inatividade física e o tempo em frente às telas da TV e do computador. Estudo realizado com adolescentes espanhóis demonstrou que o tempo prolongado em frente à TV esteve associado com níveis mais baixos de colesterol HDL. ${ }^{25}$ Zheng et al., ${ }^{9}$ em pesquisa com escolares chineses, não encontraram associação entre inatividade física com dislipidemia, porém, a atividade física em horários de lazer e horário escolar são de suma importância na prevenção e combate à dislipidemia. Moschonis et al. ${ }^{26}$ ainda defendem que a prática de 45 minutos de atividade física intensa ou moderada por dia, juntamente com cinco refeições por dia, diminui a probabilidade de crianças desenvolverem dislipidemia.

No nosso estudo, a dislipidemia também não esteve associada com o baixo consumo de frutas e hortaliças. Diferentemente, no estudo de Bradlee et al. ${ }^{27}$ nos Estados Unidos, identificou-se que meninas que consomem mais frutas e vegetais, bem como mais grãos integrais, eram menos propensas a ter níveis de 
lipídios de alto risco. No estudo de Yuan et al., ${ }^{28}$ observou-se associação inversa ao consumo regular de frutas com o hipertrigliceridemia, porém não foi encontrado nenhuma relação com o consumo de vegetais. Contudo, Takahashi et al. ${ }^{29}$ constataram que a ingestão de frutas e vegetais é considerado fator de proteção para hipergliceridemia e que o excesso de gordura central e periférica é um importante marcador de dislipedimia. Song e colaboradores ${ }^{30}$ ressaltam ainda que o consumo em excesso de carboidratos esteja diretamente ligado ao risco de desenvolver dislipidemia.

Ding e colaboradores, ${ }^{31}$ em estudo com crianças e adolescentes chineses, destacam uma preocupação entre a relação dos níveis séricos de lipídeos e dislipidemia com obesidade e seus possíveis agravamentos cardiovasculares. Casavalle et al. $^{10}$ ainda reforçam, em seu estudo com 139 crianças e adolescentes obesos, em Buenos Aires, Argentina, que indivíduos com excesso de peso tem maior propensão à desenvolver dislipidemia, bem como o excesso de peso é um importante preditor de saúde que requer preocupação. ${ }^{10}$

Hovsepian et al., ${ }^{11}$ em seu estudo de revisão sobre dislipidemia em crianças iranianas, constataram que dentre as crianças com dislipidemia, os indicadores principais foram a hipertrigliceridemia e baixo $\mathrm{HDL}$, priorizando intervenções futuras, visando estes aspectos. Contudo, Elmaoğulları et al. ${ }^{12}$ ainda reforçam que a prevalência de dislipidemia em obesos é alta e se eleva com a idade; em alguns casos, pode gerar agravamentos como resistência à insulina e hepatoesteatose, assim sugerindo maior propensão à desenvolver complicações clínicas e metabólicas, que devem ser acompanhados de perto.

\section{CONSIDERAÇÕES FINAIS}

A dislipidemia mostrou associação com variáveis sociodemográficas, sendo mais prevalente na região centro e em escolas da rede pública (municipal e estadual). Por outro lado, os hábitos sedentários e o pouco consumo de frutas/verduras não estiveram associados. Porém, é importante ressaltar a alta prevalência de dislipidemia nos escolares avaliados. A identificação das variáveis associadas pode auxiliar na elaboração de estratégias de saúde pública.

\section{REFERÊNCIAS}

1. Mello ED, Luft VC, Meyer F. Obesidade Infantil: como podemos ser eficazes? J Pediatr (Rio J.) 2004;80(3):173-182.

2. Santos PT, Bertolozzi MR, Hino P. Necessidades de saúde na atenção primária: percepção de profissionais que atuam na educação permanente. Acta Paul Enferm 2010;23(6):788-9

3. FrancaE, Alves JGB. Dislipidemiaentre criançase adolescentes de Pernambuco. Arq Bras Cardiol 2006;87(6):722-727.

4. Sichieri R, Souza RA. Estratégia para prevenção da obesidade em crianças e adolescentes. Cad Saúde Pública 2008;24(2):209-223.

5. Ramos AT, Carvalho DF, Gonzaga NC, Cardoso AS, Noronha JAF, Cardoso MAA. Perfil lipídico em crianças e adolescentes com excesso de peso. Rev Bras Crescimento Desenvolv Hum 2011;:21(3):780-788.

6. Carvalho EAA, Simão MTJ, Fonseca MC, Andrade
RG, Ferreira MSG, Silva MF, Souza IPR, Fernandes BS. Obesidade aspecto epidemiológicos e prevenção. Rev Medica Minas Gerais 2013;23(1):74-82. doi: 10.5935/22383182.20130012

7. Lachtermacher R. Dislipidemia no adolescente: fator de risco de aterosclerose na idade adulta? Quando pesquisar. Adolescência \& Saúde 2004;1(1):31-36.

8. Pizzi J, Silva LR, Moser D, Leite N. Relação entre aterosclerose subclínica, pressão arterial e perfil lipídico em crianças e adolescentes obesos: uma revisão sistêmica. Arq Bras Endocrinol Metabol 2013;57(1):1-6.

9. Zheng W, Chen Y, Zhao A, Xue Y, Zheng Y, Mu Z, Wang P, Zhang Y. Associations of sedentary behavior and physical activity with physical measurements and dyslipidemia in school-age children: a cross-sectional study. BMC Public Health 2016;16:1186. doi 10.1186/s12889-016-3826-y

10. Casavalle $P$ L, Lifshitz F, Romano LS, Pandolfo $M$, Caamaño A, Boyer PM, Rodríguez PN, Friedman SM. Prevalence of dyslipidemia and metabolic syndrome risk factor in overweight and obese children. Pediatric Endocrinology Reviews 2014;12(2):213-223.

11. Hosvsepian S, Kelishadi R, Djalalinia S, Farzadfar F, Naderimagham S, Qorbani M. Prevalence of dyslipidemia in Iranian children and adolescents: A systematic review. Journal of Research in Medical Sciences : The Official Journal of Isfahan University of Medical Sciences 2015;20(5):503-521.

12. Elmaoğulları S, Tepe D, Uçaktürk SA, Kara FK, Demirel F. Prevalence of dyslipidemia and associated factors in obese children and adolescents. J Clin Res Pediatr Endocrinol 2015;7(3):228-234. doi: 10.4274/jcrpe.1867

13. Pires A, Sena C, Seiça R. Dyslipidemia and cardiovascular changes in children. Current Opinion in Cardiology 2016;31(1):95-100. doi: 10.1097/ HCO.0000000000000249

14. Raj M. Cardiovascular health in children and adolescents. A Journal of Cardiological Society of India, Kerala Chapter 2014;4(2):22-28.

15. Friedwald WT, Levy RI, Fredrickson DS. Estimation of the concentration of low-density lipoprotein cholesterol in plasma, without use of the preparative ultracentrifuge. Clinical Chemistry 1972;18(6):499-502.

16. NHLBI. National Heart, Lung, and Blood Institute. Expert panel on integrated guidelines for cardiovascular health and risk reduction in children and adolescents. Bethesda: National Heart, Lung, and Blood Institute, 2012.

17. Burgos MS et al. Saúde dos escolares - Fase III. Avaliação de indicadores bioquímicos, genéticos, hematológicos, imunológicos, posturais, somatomotores, saúde bucal, fatores de risco às doenças cardiovasculares e estilo de vida de escolares: estudo em Santa Cruz do Sul-RS (Projeto de Pesquisa). Santa Cruz do Sul: UNISC, 2014.

18. AAP. American Academy of Pediatrics. Children, adolescents, and television. Pediatrics 2001;107(2):423-426.

19. PROESP-BR - Projeto Esporte Brasil. Manual. 2009. Disponível em: http://www.proesp.ufrgs.br. Acesso em: 13 abr. 2015.

20. Barros MVG, Nahas MV. Medidas da atividade física: teoria e aplicação em diversos grupos populacionais. Londrina: Midiograf, 2003.

21. Neto ODA, Silva RCR, Assis AMO, Pinto, EJ. Fatores associados á dislipidemia em crianças e adolescentes de escolas públicas de Salvador, Bahia. Rev Bras de Epid 2012;15(2):335-345. 
22. Ribas SA, Silva LCS. Fatores de risco cardiovascular e fatores associados em escolares do município de Belém, Pará, Brasil. Cader de Sau Púb 2014;30(3):577-586. doi: 10.1590/0102 $311 \times 00129812$

23. Van Den Berg G, Eijsden MV, Vrijkotte TGM, Gemke RJBJ. Socioeconomic inequalities in lipid and glucose metabolism in early childhood in a population - based cohort: The ABCDstudy. BMC Public Health 2012;12(591):1-8.

24. Silva D A, Franceschini SCC, Novaes JF, Santos RHS, Priore SE. Dyslipidemias and related factors in brazilian adolescents from rural and urban areas. Health 2014;6(11):1191-1200. doi:10.4236/health. 2014.611146

25. Martinez-Gomez D, Rey-López P, Chillón P, Gómez-Martínez $\mathrm{S}$, Vicente-Rodríguez G, Martín-Matillas M, Garcia-Fuentes M, Delgado M, Moreno LA, Veiga OL, Eisenmann JC, Marcos A. Excessive TV viewing and cardiovascular disease risk factors in adolescents. The AVENA cross- sectional study. BMC Public Healt 2010;10(274):1-8. doi: 10.1186/1471 2458-10-274

26. Moschonis G, Mavrogianni C, Karatzi K, latridi V, Chrousos GP, Lionis $\mathrm{C}$, Manios Y. Increased physical activity combined with more eating occasions is beneficial against dyslipidemias in children. The healthy growth study. Eur J Nutr 2013;52: 1135. doi: 10.1007/s00394-012-0424-3
27. Bradlee ML, Singera MR, Danielsb SR, Moorea LL. Eating patterns and lipid levels in older adolescente girls. Nutrition, metalolism \& cardiovascular diseases 2013;23(3):196-204. doi: 10.1016/j.numecd.2011.10.010.

28. Yuan C, Lee HJ, Shin HJ, Stampfer MJ, Cho E. Fruit and vegetable consumption and hypertriglyceridemia: korean national health and nutrition examination surveys (knhanes) 2007-2009. European Journal of Clinical Nutrition 2015;69:1193-1199. doi: 10.1038/ejcn.2015.77.

29. Takahashi M M, Oliveira EP, Moreto F, Portero-McLellan KC, Burini RC. Association of dyslipidemia with intakes of fruit and vegetables and the body fat content of adults clinically selected for a lifestyle modification program. Archivos Latinoamericanos de Nutrición 2010;60(2):148.

30. Song S J, Lee JE, Paik HY, Park MS, Song YJ. Dietary patterns based on carbohydrate nutrition are associated with the risk for diabetes and dyslipidemia. Nutr Res Pract 2012;6(4):349-356. doi: 10.4162/nrp.2012.6.4.349

31. Ding W, Cheng H, Yan Y, Zhao X, Chen F, Huang G, Hou D, Mi1 J. 10-Year trends in serum lipid levels and dyslipidemia among children and adolescents from several schools in Beijing, China. J Epidemiol 2016;26(12):637-645. doi: 10.2188/jea.JE20140252

Como citar: CORREAA, Janaína Domingues et al. Associação entre dislipidemia, dados sociodemográficos, hábitos sedentários e alimentação inadequada em escolares do sul do Brasil. Cinergis, Santa Cruz do Sul, v. 18, n. 2, mar. 2017. ISSN 2177-4005. Disponível em: <https://online.unisc.br/seer/index.php/cinergis/article/view/9023>. Acesso em: 29 mar. 2017. doi: http://dx.doi. org/10.17058/cinergis. v18i2.9023. 